\title{
New species of lichenicolous fungi for Ukraine
}

\author{
Alexander Ye. Khodosovtsev \& Valeriy V. Darmostuk \\ Kherson State University, 27, 40 Rokiv Zhovtnya str., Kherson 73000, Ukraine \\ E-mail: khodosovtsev@i.ua
}

\begin{abstract}
The lichenicolous fungi Abrothallus teloschistis, Ceratobasidium bulbillifaciens, Cladosporium licheniphilum, Cornutispora ciliata, Epicladonia sandstedei, Lichenohendersonia varians, Lichenothelia renobalesiana, Stigmidium clauzadei, Vonauxiella verrucosa, Zwackhiomyces berengerianus are reported for the first time for Ukraine. Descriptions, localities, ecology and distribution of the recorded species are provided.
\end{abstract}

Keywords: new records, Ascomycota, Basidiomycota, Eastern Europe

\section{INTRODUCTION}

Southern Ukraine includes plain and mountain territories covered by different types of landscapes. There are Festuca-Stipa and Artemisia-steppes, steppes with limestone, siliceous and loesses outcrops, sand dunes and maritime splits, small nature and artificial forests in plain part. The mountain landscapes are presented by the Crimean Mountains and Southern Crimean coastal with submediterranean climate. These landscapes are important lichen habitats, and therefore they also provide conditions for lichenicolous fungi. The new species Pronectria caloplacae Khodos. et al., P. diplococca Kocourk. et al. (Khodosovtsev et al., 2012) and Pleospora xanthoriae Khodos. \& Darmostuk (Khodosovtsev, Darmostuk, 2016) were described here. Several new Ukrainian records of lichenicolous fungi have been published from these landscapes during the last years (Khodosovtsev, 2013; Khodosovtsev et al., 2013; Khodosovtsev \& Klymenko, 2015; Darmostuk, 2015; Naumovych \& Darmostuk, 2015). The information about locations of ten new for Ukraine lichenicolous fungi which were collected mainly from the southern part of the country are presented in this contribution.

\section{MATERIAL AND METHODS}

Specimens were examined using standard microscope techniques and LOMO microscopes MBS-2 and MICROMED-2. Microscopical examination was done in water, $10 \% \mathrm{KOH}(\mathrm{K})$, Lugol's iodine, directly (I) or after a $\mathrm{KOH}$ pretreatment $(\mathrm{K} / \mathrm{I})$ or Brilliant Cresyl blue $(\mathrm{BCr})$. Photographs were taken with camera Levenhuk
C510 NG. All examined specimens are deposited in the lichenological herbarium of Kherson State University (KHER). In distribution data for all species only the first record in each country is noted.

\section{THE SPECIES}

ABrothallus teloschistis Brackel, Pérez-Ortega $\&$ Suija

Specimen examined. Ukraine, Autonomous Republic of Crimea, Arbatskaya Strelka, near the fortress Arabat, $45^{\circ} 17^{\prime} 43.64^{\prime \prime} \mathrm{N}, 35^{\circ} 28^{\prime} 21.23^{\prime \prime} \mathrm{E}$, alt. $1 \mathrm{~m}$, on thallus of Seirophora lacunosa which was infected also by Lichenoconium erodens, 10 June 2003, A. Khodosovtsev (KHER 9309).

Notes - Recently described from Europe (Italy) and North America (Cuba) (Brackel, 2015). New to Eastern Europe.

Ceratobasidium bulbillifaciens Diederich \& Lawrey (Fig. 1A).

Specimens examined. Ukraine, Kherson region, Goloprystanskiy district, village Burkuty, $46^{\circ} 22^{\prime} 02.9^{\prime \prime} \mathrm{N}$, $32^{\circ} 46^{\prime} 29.7^{\prime \prime} \mathrm{E}$, alt. $26 \mathrm{~m}$, on thalli of Xanthoria parietina, on Populus tremula, 9 April 2008, A. Khodosovtsev (KHER 9507); Velykooleksandrivkiy district, village Mala Oleksandrivka, right bank of river Ingulets, $47^{\circ} 17^{\prime} 38.47^{\prime \prime N}, 33^{\circ} 16^{\prime} 24.21^{\prime \prime} \mathrm{E}$, alt. $21 \mathrm{~m}$, on Circinaria calcarea, on limestone, 3 July 2015, V. Darmostuk (KHER 9483); Mykolayiv region, Bashtanskiy district, village Maryivka, $47^{\circ} 10^{\prime} 22.79^{\prime \prime} \mathrm{N}, 32^{\circ} 14^{\prime} 56.44^{\prime \prime} \mathrm{E}$, alt. $15 \mathrm{~m}$, on Lasallia pustulata, on granite, 8 July 2004, I. Moysiyenko (KHER 1387).

Notes - Widespread in Western and Central Europe: Belgium, France, Germany, Luxembourg, the Netherlands and Sweden (Diederich et al., 2014b). New to Eastern Europe. 
Cladosporium Licheniphilum Heuchert \& U. Braun Specimens examined. Ukraine, Kherson region, Goloprystanskiy district, village Kardashynka, $46^{\circ} 33^{\prime} 16.03$ 'N, $32^{\circ} 37^{\prime} 13.17$ 'E, alt. $3 \mathrm{~m}$, on thallus of Xanthoria parietina, on Armeniaca vulgaris, 6 January 2015, A. Khodosovtsev (KHER 9501); Tsurupinskiy district, village Proletarka, 46 38'49.7'N, 32 ${ }^{\circ} 59^{\prime} 37.1$ 'E, alt. $13 \mathrm{~m}$, on thallus of Xanthoria parietina, on Populus tremula, 5 April 2008, A. Khodosovtsev (KHER 6187); Goloprystanskiy district, village Promin, near lake Shelemens 'ke, 46²0'15”N, 3249'07'E, alt. 26 m, on Xanthoria parietina, on Populus tremula, 21 November 2015, A. Khodosovtsev \& V. Darmostuk (KHER 9430).

Notes - Known on various lichens in Europe: Estonia (Suija et al., 2011), Germany (Brackel, 2009), Italy (Brackel, 2008), Lithuania (Motiejūnaitè et al., 2012), Norway (Brackel, 2009); Asia: India (Zhurbenko, 2013a), Russia (Heuchert \& Braun, 2006).

\section{CORNUTISPORA CILIATA Kalb}

Specimens examined. Ukraine, Kherson region, Berislavskiy district, Kamyanskiy beam, $47^{\circ} 03^{\prime} 31.90^{\prime \prime} \mathrm{N}$, $33^{\circ} 35^{\prime} 11.50$ ”E, alt. $35 \mathrm{~m}$, on apothecia of Xanthoria parietina together with Lichenoconium xanthoriae M.S. Christ., on Robinia pseudoacacia, 30 September 2015, V. Darmostuk (KHER 9504).

Notes - Recorded from Europe: Austria (Berger et al., 1998), Belgium (Diederich et al., 2014a), Germany (Brackel \& Kocourková, 2006), Luxembourg (van den Boom et al., 1996), the Netherlands (van den Boom, 2002), Norway (Alstrup et al., 2008), Poland (Kukwa \& Flakus, 2009), Spain (Etayo, 1996), Sweden (Thell et al., 2014); Asia: Japan (Zhurbenko et al., 2015), Russia (Zhurbenko, 2012); Africa: (Spain), Canary Islands (Hafellner, 1996); North America: Canada (Cole \& Hawksworth, 2001), USA (Gierl \& Kalb, 1993); South America: Chile (Etayo \& Sancho, 2008); Australia: Tasmania (Gierl \& Kalb, 1993), New Zealand (Kalb et al., 1995). New to Eastern Europe.

Epicladonia sandstedei (Zopf) D. Hawksw.

Specimen examined: Ukraine, Kherson region, Velykooleksandrivkiy district, village Mala Oleksandrivka, Rusova beam, $47^{\circ} 16^{\prime} 15.93$ 'N, $33^{\circ} 14^{\prime} 05.43^{\prime \prime} \mathrm{E}$, alt. $37 \mathrm{~m}$, on thallus of Cladonia foliacea, on soil, 10 January 2016, V. Darmostuk (KHER 9512).

Notes - Known from Europe: Austria (Mayrhofer et al., 1989), Belarus (Tsurykau et al., 2014), Belgium (Diederich et al., 1991), Czech Republic (Bachmann, 1927), Denmark (Alstrup \& Svane, 1998), Estonia (Suija et al., 2010), France (Hawksworth, 1981), Germany (Sandstede,
1906), Great Britain (Hawksworth, 1981), Italy (Brackel, 2013), Lithuania (Motiejūnaite, 1999), the Netherlands (Brand et al., 2013), Norway (Sandstede, 1931), Poland (Czyżewska et al., 2005), Russia (Zhurbenko \& Himelbrant, 2002), Spain (Alvarez \& Carballal, 1992), Sweden (Hawksworth, 1981), Switzerland (Hawksworth, 1981); Asia: Russia (Zhurbenko, 1998); North America: Canada (Scholz, 1998), USA (Cole \& Hawksworth, 2001); South America: Chile (Diederich, 2003); Faeroe Islands (Alstrup \& Christensen, 1999).

LICHENOHENDERSONIA VARIANS Calatayud \& Etayo (Fig. 1C).

Specimen examined: Ukraine, Zaporizka region, Pryazovs'kiy district, village Makivka, $47^{\circ} 01^{\prime} 17.35^{\prime} \mathrm{N}$, $35^{\circ} 56^{\prime} 51.96^{\prime \prime} \mathrm{E}$, alt. $67 \mathrm{~m}$, on thallus of Candelariella vitellina, on exposed bark of Robinia near siliceous rocks outcrops, 8 October 2010, A. Khodosovtsev \& T. Zavyalova (KHER 6293).

Notes - Known from few localities in Spain (Calatayud \& Etayo, 2001) growing on saxicolous lichens such as Acarospora epithallina H. Magn., A. hilaris (Dufour) Arnold, Rhizoplaca chrysoleuca (Sm.) Zopf and Lecanora valesiaca (Müll. Arg.) Stizenb. Candelariella vitellina is a new host species. New to Eastern Europe.

Lichenothelia RENobalesiana D. Hawksw. \& V. Atienza (Fig. 1B).

Specimens examined. Ukraine, Autonomous Republic of Crimea, Simferopolskiy region, Mt Chatyrdag, lower plateau, $44^{\circ} 44^{\prime} 17.68^{\prime \prime} \mathrm{N}, 34^{\circ} 17^{\prime} 34.06^{\prime \prime} \mathrm{E}$, alt. 900 m, on Bagliettoa sp., on limestone, 2 October 1999, A. Khodosovtsev (KHER 7006); Sudakskiy region, Sudak, opposite the tourist base "Horyzont", 4449'46.90"N, $34^{\circ} 54^{\prime} 51.08$ 'E, alt. $100 \mathrm{~m}$, on Bagliettoa sp., on limestone, 22 July 1999, A. Khodosovtsev (KHER 0858).

Notes - Known from Europe: Austria (Atienza \& Hawksworth, 2008), Belgium (Atienza \& Hawksworth, 2008), Estonia (Martin et al., 2012), France (Atienza \& Hawksworth, 2008), Germany (Atienza \& Hawksworth, 2008), Great Britain (Atienza \& Hawksworth, 2008), Slovenia (Atienza \& Hawksworth, 2008), Spain (Atienza \& Hawksworth, 2008); Asia: Russia (Urbanavichus et al., 2011); Africa: Morocco (Atienza \& Hawksworth, 2008); North America: USA (Kocourková \& Knudsen, 2009).

Pestalotiopsis sp. (Fig. 2).

Specimen examined. Ukraine, Autonomous Republic of Crimea, Sudakskiy district, cape Meganom, $44^{\circ} 47^{\prime} 41.48^{\prime \prime} \mathrm{N}, 35^{\circ} 04^{\prime} 39.56^{\prime \prime} \mathrm{E}$, alt. $63 \mathrm{~m}$, on thallus 

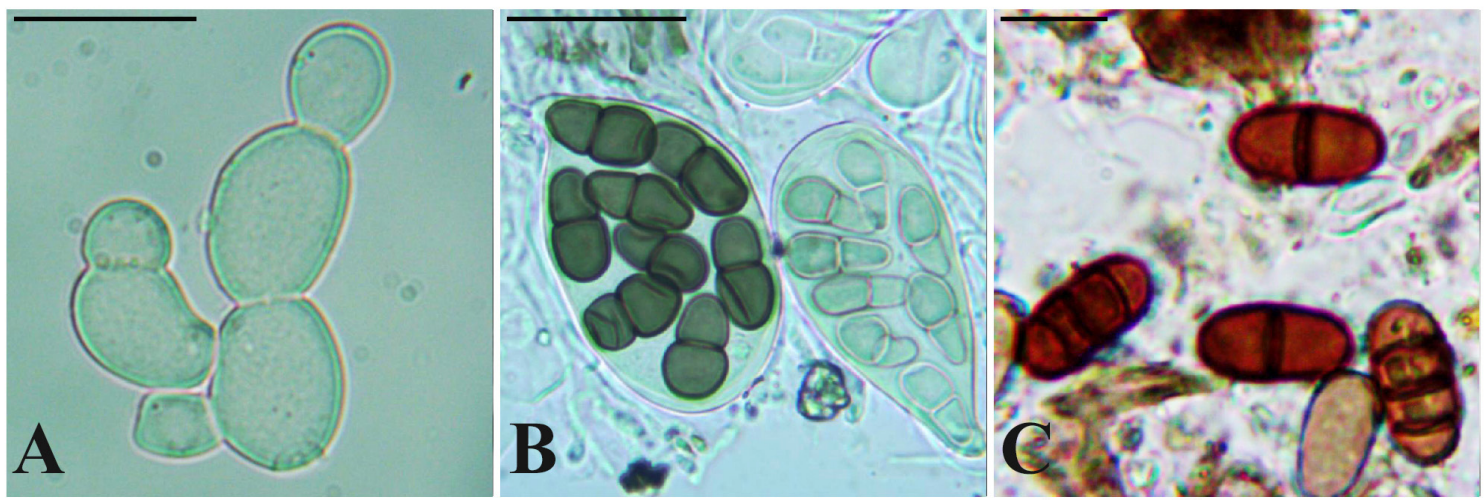

Fig. 1. A - hyphal cells in squash bulbil of Ceratobasidium bulbillifaciens; B - asci and ascospores of Lichenothelia renobalesiana; $\mathrm{C}$ - conidia of Lichenohendersonia varians (all in water). Scale bars: A - $25 \mu \mathrm{m}$; B - $50 \mu \mathrm{m}$; C - $10 \mu \mathrm{m}$.

of Squamarina lentigera, on soil, 2 May 2002, A. Khodosovtsev (KHER 6957).

Notes - Only few pycnidia were present, 150-200 $\mu \mathrm{m}$ diam., immersed in lobes of the host. Conidiogenous cells ampulliform or lageniform, hyaline. Conidia fucoid, ellipsoid, straight, 4septate, 20-26 × 9.5-14.5 $\mu \mathrm{m}$, basal cell obconic, hyaline; apical cell 3-5 $\mu \mathrm{m}$ long, hyaline, subcylindrical, with 3 tubular apical appendages, arising from an apical crest, unbranched, filiform, 30-50 $\mu \mathrm{m}$ long, basal appendage single, tubular, short, unbranched, centric, 2-3 $\mu \mathrm{m}$ long.

Pestalotiopsis is generally not a lichenicolous genus, but Pestalotiopsis maculans (Corda) Nag Raj was cultured from some North American lichens (Sun et al., 2002) and Pestalotiopsis sp. was found in Dirinaria picta (Suryanarayanan et al., 2005). Pestalotiopsis is considered en- dolichenic in both above mentioned cases. Our specimen has wide conidia $(>10 \mu \mathrm{m})$ which is unknown in Pestalotiopsis species so far (Maharachchikumura et al., 2014).

Stigmidium Clauzadei Cl. Roux \& Nav.-Ros.

Specimen examined. Ukraine, Kherson region, Bilozerskiy district, village Fedorivka, 4648'19.17'N, $32^{\circ} 47^{\prime} 52.55^{\prime} \mathrm{E}$, alt. $10 \mathrm{~m}$, on thallus of Verrucaria viridula, on limestone, 25 May 1995, A. Khodosovtsev (KHER 9511).

Notes - Reported from Europe: Denmark (Søchting et al., 2007), Estonia (Suija et al., 2011), France (Roux \& Navarro-Rosines 1994), Germany (Brackel, 2009), Great Britain (Hitch, 2007), Luxembourg (Sérusiaux et al., 1999), the Netherlands (Brand et al., 2013), Poland (Kukwa \& Czarnota, 2006) and Spain (Roux \& Navarro-
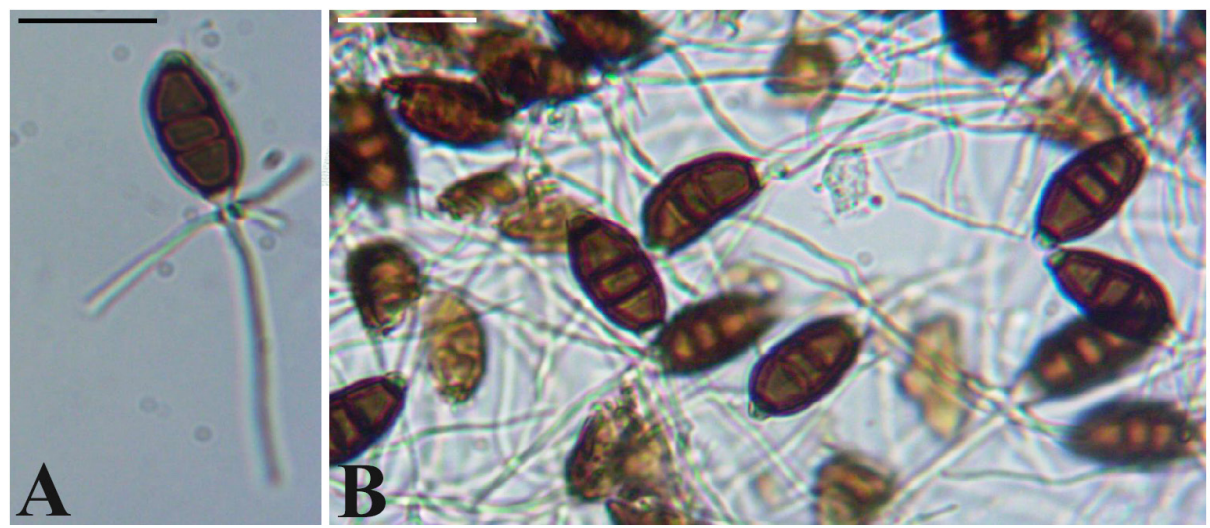

Fig. 2. Pestalotiopsis sp.: A, B - conidia. Scale bars: A, B - $25 \mu \mathrm{m}$. 
Rosines, 1994); Asia: Russia (Urbanavichus \& Urbanavichene, 2015).

Vouauxiella Verrucosa (Vouaux) Petr. \& Syd. Specimen examined. Ukraine, Autonomous Republic of Crimea, Sudakskiy district, village Novyy Svit, $44^{\circ} 49^{\prime} 29.46$ "N, $34^{\circ} 54^{\prime} 30.26^{\prime \prime} \mathrm{E}$, alt. $61 \mathrm{~m}$, on apothecia of Lecanora cfr. allophana, on bark of Juniperus excelsa, 9 July 2001, A. Khodosovtsev (KHER 6769, 9355).

Notes - Known from Europe: Austria (Türk \& Wittmann, 1987), 1981), France (Bouly de Lesdain, 1907), Germany (Triebel \& Scholz, 2001), Great Britain (Hawksworth, Greece (Hawksworth, 1981), Italy (Brackel, 2011), Portugal (Hafellner, 1995), Spain (Hawksworth, 1981), Sweden (Hawksworth, 1981); Asia: Pakistan (Hawksworth, 1981); Africa: Morocco (Hafellner, 1996); North America: USA (Diederich, 2003); Azorean archipelago (Berger, Priemetzhofer, 2008). New to Eastern Europe.

\section{ZwaCKHIOMYCES Berengerianus (Arnold) Grube \& Triebel}

Specimen examined. Ukraine, Odessa region, Kominternovskiy district, village Serbky, $47^{\circ} 03^{\prime} 27.83^{\prime \prime} \mathrm{N}$, $30^{\circ} 57^{\prime} 58.57^{\prime} \mathrm{E}$, alt. $27 \mathrm{~m}$, on Mycobilimbia sabuletorum, on limestone, 2 May 1996, A. Khodosovtsev (KHER 9648); Zakarpatska region, Carpathian Biosphere Reserve, near river Velyka Uholka, $48^{\circ} 13^{\prime} 19.37^{\prime \prime N}, 23^{\circ} 42^{\prime} 39.16^{\prime \prime E}$, alt. $814 \mathrm{~m}$, on $\mathrm{Myco}-$ bilimbia sabuletorum above limestone, 15 July 2010, O. Nadyeina (KHER 9528).

Notes - Known from Europe: Austria (Arnold, 1871), France (Grube \& Hafellner, 1990), Germany (Lettau, 1958), Great Britain (Grube \& Hafellner, 1990), Italy (Hawksworth, 1983), Netherlands (Brand et al., 2013), Norway (Zhurbenko \& Brackel, 2013), Poland (Kukwa \& Flakus, 2009), Russia (Zhurbenko, 2004), Sweden (Grube \& Hafellner, 1990); Asia: Russia (Zhurbenko \& Santesson, 1996); North America: Canada (Zhurbenko, 2013b), USA (Zhurbenko et al., 1995).

\section{ACKNOWLEDGEMENTS}

We wish to thank Dr. M. Zhurbenko for valuable corrections of the manuscript and kindly providing literature, Dr. O. Nadyeina who generously provided the specimen of Mycobylimbia sabuletorum for study and Dr. W. Brackel for kindly provided literature. We are grateful to Dr. J. Vondrák for some linguistic corrections.

\section{REFERENCES}

Alstrup, V. \& Christensen, S. N. 1992. The NLF-excursion to the Faeroe Islands 1995. Graphis Scripta 8: 25-29.

Alstrup, V., \& Svane, S. 1998. Interesting lichens and lichenicolous fungi from northeast Jutland, Denmark. Graphis Scripta 9(1): 23-25.

Alstrup, V., Grube, M., Motiejūnaitè, J., Nordin, A. \& Zhurbenko, M. 2008. Lichenicolous fungi from the Skibotn area, Troms, Norway. Graphis Scripta 20: $1-8$.

Alvarez, J. \& Carballal, R. 1992. Liquenes y hongos liquenicolas interesantes de la Sierra de Caurel (Lugo, noroeste de España). Cryptogamie. Bryologie, lichenologie 14: 303-320.

Arnold, F. 1871. Lichenologische Ausfltige in Tirol 6. Die Waldrast. Verhandlungen der ZoologischBotanischen Gesellschaft in Wien 21: 1103-1148.

Atienza, V. \& Hawksworth, D. L. 2008. Lichenothelia renobalesiana sp. nov. (Lichenotheliaceae), for a lichenicolous ascomycete confused with Polycoccum opulentum (Dacampiaceae). The Lichenologist 40: 87-96. http://dx.doi.org/10.1017/ S0024282908007342

Bachmann, E. 1927. Das Verhältnis flechtenbewohnender Pilze zu ihren Wirtspflanzen. Archiv für Protistenkunde 58: 143-172.

Berger, F. \& Priemetzhofer, F. 2008. Neufunde und interessante Nachweise von Flechten und flechtenbewohnenden Pilzen von den Azoren. Herzogia 21: 125-146.

Berger, F., Priemetzhofer, F. \& Türk, R. 1998. Neue und seltene Flechten und Iiehenicole Pilze aus Oberösterreich, Österreich IV. Beiträge zur Naturkunde OberästetTeichs 6: 397-416.

Bouly de Lesdain, 1907. Lichens des environs de Versailles. Bulletin de la Société botanique de France 54: 680-698.

Brackel, W. von. 2008. Zwackhiomyces echinulatus sp. nov. and other lichenicolous fungi from Sicily, Italy. Herzogia 21: 181-198.

Brackel, W. von. 2009. Flechtenbewohnende Pilze in Bayern IV. Berichte der Bayerischen Botanischen Gesellschaft 79: 5-55.

Brackel, W. von. 2011. Lichenicolous fungi and lichens from Puglia and Basilicata (southern Italy). Herzogia 24: 65-101. http://dx.doi.org/10.13158/ heia.24.1.2011.65

Brackel, W. v. 2013. Miscellaneous records of lichenicolous fungi from the Italian Alps. Herzogia 26: 141-157. http://dx.doi.org/10.13158/ heia.26.1.2013.141

Brackel, W. von. 2015. Lichenicolous fungi from Central Italy with notes on some remarkable hepaticolous, algicolous and lichenized fungi. Herzogia 28: 212-281. http://dx.doi.org/10.13158/ heia.28.1.2015.212

Brackel, W. von. \& Kocourková, J. 2006. Endococcus karlstadtensis sp. nov. und weitere Funde von 
flechtenbewohnenden Pilzen in Bayern-Beitrag zu einer Checkliste II. Berichte der Bayerischen Botanischen Gesellschaft 76: 5-32.

Brand, A. M., Sparrius, L. B. \& Aptroot, A. 2013. Nieuwe soorten korstmossen en lichenicole fungi voor Nederland. Buxbaumiella 97: 17-22.

Calatayud, V. \& Etayo, J. 2001. Five new species of lichenicolous conidial fungi from Spain. Canadian Journal of Botany 79: 223-230. http://dx.doi. org/10.1139/b00-144

Cole, M. S. \& Hawksworth, D. L. 2001. Lichenicolous fungi, mainly from the USA, including Patriciomyces gen. nov. Mycotaxon 77: 305-338.

Czyżewska, K., Motiejūnaitè, J. \& Cieśliński, S. 2005. New and noteworthy species of lichens and allied fungi from North-Eastern Poland. Acta Mycologica 40: 277-291. http://dx.doi.org/10.5586/ am.2005.025

Darmostuk, V. V. 2015. Codonmyces lecanorae Calat. $\&$ Etayo is a new species of lichenicolous fungi for Ukraine. (In Ukrainian). Chornomorski Botanical Journal 11: 327-329.

Diederich, P. 2003. New species and new records of American lichenicolous fungi. Herzogia 16: 41-90.

Diederich, P., Sérusiaux, E. \& van den Boom, P. 1991. Lichens et champignons lichénicoles nouveaux ou intéressants pour la flore de Belgique et des régions voisines. V. Lejeunia nouvelle série 136: $1-47$.

Diederich, P., Ertz, D., Eichler, M., Cezanne, R., van den Boom, P., van den Broeck, D. \& Sérusiaux, E. 2014a. New or interesting lichens and lichenicolous fungi from Belgium, Luxembourg and northern France. XV. Bulletin de la Société des naturalistes luxembourgeois 115: 157-165.

Diederich, P., Lawrey, J. D., Capdet, M., Pereira, S., Romero, A., Etayo, J., Flakus, A., Sikaroodi, M. \& Ertz, D. 2014b. New lichen-associated bulbilforming species of Cantharellales (Basidiomycetes). The Lichenologist 46: 333-347. http:/ / dx.doi. org/ 10.1017/S0024282913000583

Etayo, J. 1996. Aportación a la flora liquénica de las Islas Canarias. I. Hongos liquenícolas de Gomera. Bulletin de la Société Linnéenne de Provence 47: 93-110.

Etayo, J. \& Sancho, L. G. 2008. Hongos liquenicolas del Sur de Sudamerica, especialmente de Isla Navarino (Chile). Bibliotheca Lichenologica 98: 1-302.

Gierl, C. \& Kalb, K. 1993. Die Flechtengattung Dibaeis. Eine Ubersicht uber die rosafruchtigen Arten von Baeomyces sens. lat. nebst Anmerkungen zu Phyllobaeis gen. nov. Herzogia 9: 593-645.

Grube, M. \& Hafellner, J. 1990. Studien an flechtenbewohnenden Pilzen der Sammelgattung Didymella (Ascomycetes, Dothideales). Nova Hedwigia 51: 283-360.

Hafellner, J. 1995. A new checklist of lichens and lichenicolous fungi of insular Laurimacaronesia including a lichenological bibliography for the area. Fritschiana 5: 1-132.
Hafellner, J. 1996. Bemerkenswerte Funde von Flechten und lichenicole Pilze auf makaronesischen Inseln IV. Einige bisher libersehene lichenicole Arten der kanarischen Inseln. Cryptogamie. Bryologie et Lichenologie, 17: 1-14.

Hawksworth, D. L. 1981. The lichenicolous Coelomycetes. Bulletin of the British Museum (Natural History). Botany series 9: 1-98.

Hawksworth, D. L. 1983. A key to the lichen-forming, parasitic, parasymbiotic and saprophytic fungi occurring on lichens in the British Isles. The Lichenologist 15: 1-44. http:/ /dx.doi.org/10.1017/ S0024282983000031

Heuchert, B. \& Braun, U. 2006. On some dematiaceous lichenicolous hyphomycetes. Herzogia 19: 11-21.

Hitch, C. J. B. (ed.) 2007. New, rare and interesting lichens. British Lichen Society Bulletin 101: 67-86.

Kalb, K., Hafellner, J. \& Staiger, B. 1995. Lichenicole Pilze auf Arten der Flechtengattung Haematomma. Bibliotheca Lichenologica 59: 199-222.

Khodosovtsev, A. 2013. Lichen-forming and lichenicolous fungi from Aju-Dag Mt new to Ukraine and Crimea. (In Ukrainian). Chornomorski Botanical Journal 9: 84-88.

Khodosovtsev, A. Ye., Darmostuk, V. V. 2016. Pleospora xanthoriae sp. nov. (Pleosporaceae, Pleosporales), a new lichenicolous fungus on Xanthoria parietina from Ukraine, with a key to the known lichenicolous species of Dacampia and Pleospora. Opuscula Philolichenum 15: 6-11.

Khodosovtsev, A., Dymytrova, L., Nadyeina, A., Naumovych, A., Khodosovtseva, Yu. \& Scheidegger, C. 2013. A contribution to beech forest-associated epiphytic lichen-forming and lichenicolous fungi in Crimean Mts (Ukraine). Flora Mediterranea 23: $57-68$.

Khodosovtsev, A. Ye. \& Klymenko, V. M. 2015. Didymellopsis perigena (Nyl.) Grube and Zwackhiomyces cervinae Calat., Triebel \& Pérez-Ortega (Xanthopyreniaceae, Ascomycota) new for Ukraine species of the lichenicolous fungi. (In Ukrainian). Chornomorski Botanical Journal 11: 217-222.

Khodosovtsev, A., Vondrák, J., Naumovich, A. Kocourková, J., Vondrákova, O. \& Motiejūnaitė, J. 2012. Three new Pronectria species in terricolous and saxicolous microlichen communities (Bionectriaceae, Ascomycota). Nova Hedwigia 95: 211-220. http://dx.doi.org/10.1127/00295035/2012/0026

Kocourková, J. \& Knudsen, K. 2009. Three lichenicolous fungi new for North America. Evansia 26(3): 148-151. http:/ / dx.doi.org/10.1639/0747-985926.3.148

Kukwa, M. \& Czarnota, P. 2006. New or interesting records of lichenicolous fungi from Poland IV. Herzogia 19: 111-123.

Kukwa, M. \& Flakus, A. 2009. New or interesting records of lichenicolous fungi from Poland VII. 
Species mainly from Tatra Mountains. Herzogia 22: 191-211.

Lettau, G. 1958. Flechten aus Mitteleuropa XIII. Feddes Repert 61(1): 1-73. http://dx.doi. org/10.1002/fedr.19580610102

Maharachchikumbura, S. S. N., Hyde, K. D., Groenewald, J. Z., Xu, J. \& Crous, P. W. 2014. Pestalotiopsis revisited. Studies in Mycology 79: 121-186. http:/ /dx.doi.org/10.1016/j.simyco.2014.09.005

Martin, L., Suija, A., Schmeimann, M., Leppik, E. \& Pykälä, J. 2012. New Estonian records and amendments: Lichenized and lichenicolous fungi. Folia Cryptogamica Estonica 49: 93-96.

Mayrhofer, H., Türk, R. \& Wittmann, H. 1989. Ein Beitrag zur Flechtenflora von Vorarlberg (Österreich): Ergebnisse der Feldtagung der Bryologisch-lichenologischen Arbeitsgemeinschaft für Mitteleuropa im Juli 1986. Herzogia 8: 207-247.

Motiejūnaitè, J. 1999. Dzukijos nacionalinio parko kerpes ir su jomis susije grybai [Lichens and allied fungi of Dzukija National Park]. Botanica Lithuanica, 5: 137-153.

Motiejūnaitè, J., Berglund, T., Czarnota, P., Himelbrant, D., Högnabba, F., Konoreva, L. A., Korchikov, E. S., Kubiak, D., Kukwa, M., Kuznetsova, E., Leppik, E., Lõhmus, P., Lukošienè, I. P., Juha, P., Stončius, D., Stepanchikova, I., Suija, A., Thell, A., Tsurykau, A. \& Westberg, M. 2012. Lichens, lichenicolous and allied fungi found in Asveja Regional park (Lithuania). Botanica lithuanica 18(2): 85-100. http:/ /dx.doi.org/10.2478/ v10279-012-0011-9

Naumovych A. O., Darmostuk V. V. 2015. Lichenicolous fungi of the valley of Ingulets river (Ukraine). (In Ukrainian). Chornomorski Botanical Journal 11 (4): 512-520.

Roux, C. \& Navarro-Rosines, P. 1994. Stigmidium clauzadei sp. nov., nelikeniginta fungo likenloga (Ascomycetes). Bulletin de la Société linnéenne de Provence 45: 443-450.

Sandstede, H. 1906. Die Cladonien des nordwestdeutschen Tieflandes und der deutschen Nordseeinseln. Abhandlungen herausgegeben vom Naturwissenschaftlichen Verein zu Bremen 18: 384-457.

Sandstede, H. 1931. Die Gattung Cladonia. In Raben host's Kryptogamen-Flora von Deutschland, Österreich und der Schweiz (A. Zahlbruckner, ed). Vol. $9(4,2)$. Leipzig: Akademische Verlagsgesellschaft.

Scholz, P. 1998. Phacopsis doerfeltii, sp. nov., and two other interesting lichenicolous fungi from Canada. Sauteria 9: 37-42.

Sérusiaux, E., Diederich, P., Brand, A. M. \& van den Boom, P. 1999. New or interesting lichens and lichenicolous fungi from Belgium and Luxembourg VIII. Lejeunia 162: 1-95.

Søchting, U., Alstrup, V., Kocourková, J., Vondrak, J. \& Spiegelberg, R. 2007. Additions to the lichen and lichenicolous flora of Denmark VII. Graphis Scripta 19: 40-47.
Suija, A., Czarnota, P., Himelbrant, D., Kowalewska, A., Kukwa, M., Kuznetsova, E., Leppik E., Motiejūnaitè J., Piterāns A., Schiefelbein U., Skazina M., Sohrabi M., Stepanchikova I., \& Veres, K. 2010. The lichen biota of three nature reserves in island Saaremaa, Estonia. Folia Cryptogamica Estonica 47: 85-96

Suija, A., Leppik, E., Jüriado, I., Lõhmus, P., Marmor, L., \& Saag, L. 2011. New Estonian records and amendments: Lichenized, lichenicolous and allied fungi. Folia Cryptogamica Estonica 48: 154-158.

Sun, H. J., Depriest, P. T., Gargas, A., Rossman, A. Y. \& Friedmann, E. I. 2002. Pestalotiopsis maculans: a dominant Parasimbiont in North American Lichens. Symbiosis 33: 215-226.

Suryanarayanan, T. S., Thirunavukkarasu, N., Nariharan, G. N. \& Balaji P. 2005. Occurrence of non-obligate microfungi inside lichen thalli. Sydowia 57: 120-130.

Thell, A., Alstrup, V., Arup, U., Bendiksby, M., Czarnota, P., Feuerer, T., Haugan, R., Kärnefelt, I., Klepsland, J. T., Kukwa, M., Launis, A., Millanes, A. M., Motiejūnaitė, J., Nordin, A., Prieto, M., Pykälä, J., Seaward, M. R. D., Timdal, E., Tsurykau, A., Vitikainen, O. \& Westberg, M. 2014. New or interesting lichens and lichenicolous fungi from the Vadstena area, Östergötland, Sweden. Graphis Scripta 26: 15-33.

Triebel, D. \& Scholz, P. 2001. Lichenicolous fungi from Bavaria as represented in the Botanische Staatssammlung München. Sendtnera 7: 211-231.

Tsurykau, A., Golubkov, V. \& Kukwa, M. 2014. New or otherwise interesting records of lichens and lichenicolous fungi from Belarus. Herzogia 27(1): 111-120. http://dx.doi.org/10.13158/ heia.27.1.2014.111

Türk, R. \& Wittmann, H. 1987. Flechten im Bundesland Salzburg (Österreich) und im Berchtesgadener Land (Bayern, Deutschland) - die bisher beobachteten Arten und deren Verbreitung. Sauteria 3: 1-313.

Urbanavichus, G., Gabibova, A. \& Ismailov, A. 2011. New records of lichens and lichenicolous fungi for Russia and the Caucasus. Turkish Journal Of Botany 35: 291-297.

Urbanavichus, G. \& Urbanavichene, I. 2015. New records of lichens and lichenicolous fungi from the NW Caucasus (Russia). Herzogia 28(1): 185-192. http:/ / dx.doi.org/10.13158/heia.28.1.2015.185

van den Boom, P. 2002. Some interesting records of lichens and lichenicolous fungi from The Netherlands 5. Österreichische Zeitschrift für Pilzkunde 11: 153-157.

van den Boom, P., Diederich, P. \& Sérusiaux, E. 1996. Lichens et champignons lichénicoles nouveaux ou intéressants pour la flore de la Belgique et des régions voisines. VII. Bulletin de la Société des naturalistes luxembourgeois 97: 81-92. 
Zhurbenko, M. P. 1998. Lichens and lichenicolous fungi from the north of Pyasino lake, Taymyr Peninsula, Siberia. Folia Cryptogamica Estonica 32: 153-159.

Zhurbenko, M. P. 2004. Lichenicolous and some interesting lichenized fungi from the Northern Ural, Komi Republic of Russia. Herzogia 17: 77-86.

Zhurbenko, M. P. 2012. New records of lichenicolous fungi from State Nature Reserve "Stolby" (Krasnoyarsk territory). (In Russian). Novitates systematicae plantarum non vascularium 46: 92-95.

Zhurbenko M. P. 2013a. A first list of lichenicolous fungi from India. Mycobiota 3: 19-34. http:// dx.doi.org/10.12664/mycobiota.2013.03.03

Zhurbenko, M. P. 2013b. Lichenicolous fungi and some allied lichens from the Canadian Arctic. Opuscula Philolichenum 12: 180-197.

Zhurbenko, M. P. \& Brackel, W. von. 2013. Checklist of lichenicolous fungi and lichenicolous lichens of
Svalbard, including new species, new records and revisions. Herzogia 26: 323-359. http://dx.doi. org/10.13158/heia.26.2.2013.323

Zhurbenko, M. P. \& Himelbrant, D. E. 2002. Lichenicolous fungi from the Kandalaksha Gulf, Karelia Keretina, Russia. Folia Cryptogamica Estonica 39: 51-59.

Zhurbenko, M. P. \& Santesson, R. 1996. Lichenicolous fungi from the Russian Arctic. Herzogia 12: 147-161.

Zhurbenko, M. P., Frisch, A., Ohmura, Y. \& Thor, G. 2015. Lichenicolous fungi from Japan and Korea: new species, new records and a first synopsis for Japan. Herzogia 28: 762-789. http://dx.doi. org/10.13158/heia.28.2.2015.762

Zhurbenko, M., Santesson, R., Walker, D. A., Auerbach, N. A. \& Lewis, B. 1995. New and interesting lichenicolous fungi and lichens from Alaska. Evansia 12(3): 92-97. 
100 Folia Cryptog. Estonica 